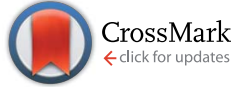

Cite this: RSC Adv., 2016, 6, 40777

\title{
Structural modification and band-gap crossover in indium selenide nanosheets $\dagger$
}

\author{
Mildred A. Airo, ${ }^{a}$ Siziwe Gqoba, ${ }^{\text {a }}$ Francis Otieno, ${ }^{\text {bc }}$ Makwena J. Moloto ${ }^{d}$ \\ and Nosipho Moloto*ab
}

Herein, we report on the synthesis of InSe nanosheets. We further report on the interdependency of concentration and time on the evolution of the nanosheets as well as the role of the capping agents on the crystal phase and morphology of the resultant particles. Our results show that hexagonal-like InSe nanosheets were synthesized at different optimum times depending on the amount of indium precursor. Regardless of the amount of indium precursor used, the product remained unchanged, i.e. InSe. This was attributed to the nature of the oleylamine capping agent. Oleylamine is known to be a reducing agent

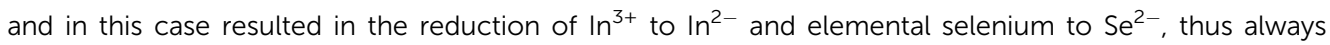
resulting in the formation of rhombohedral InSe nanosheets. A non-reducing 1-dodecanthiol cosurfactant was thus used. Dodecanthiol was thought to stabilize the $\ln ^{3+}$ ions by coordinating and forming a complex, thus preserving the $3+$ oxidation state of indium, resulting in the formation of the final product of $\mathrm{In}_{2} \mathrm{Se}_{3}$ nanocrystals. The morphology of $\mathrm{In}_{2} \mathrm{Se}_{3}$ changed depending on the amount of oleylamine used. The optical properties of the InSe were further evidence that nanosheets had been synthesized due to the band gap crossover.

Received 5th January 2016

Accepted 27th March 2016

DOI: $10.1039 / c 6 r a 00262 e$

www.rsc.org/advances

\section{Introduction}

Systematic adjustments during colloidal synthesis of reaction conditions such as time, temperature, concentration, chemistry of reagents and surfactants have been used by several researchers to manipulate the structure and morphology of nanostructures in order to attain desired chemical, optical and electronic properties based on their applications. ${ }^{1}$ In general, the nanocrystal (NC) size increases with increasing reaction time, as more material is added to the NC surfaces. ${ }^{1-6}$ For instance, in our previous studies, ${ }^{7}$ duration of synthesis was reported as an important parameter that played a role in determining the morphology and type of the colloidal InSe nanoparticles obtained, and a growth mechanism was proposed based on products obtained at different time of syntheses. Also size increases with increasing temperature, as the rate of addition of material to the existing nuclei increases. ${ }^{1}$ Tailoring

${ }^{a}$ Molecular Sciences Institute, School of Chemistry, University of the Witwatersrand, Private Bag 3, Wits, 2050, Republic of South Africa. E-mail: Nosipho.Moloto@wits. ac.za; Fax: +27 865228349; Tel: +27117176774

${ }^{b}$ Materials for Energy Research Group, University of the Witwatersrand, Private Bag 3, Wits, 2050, Republic of South Africa

'School of Physics, University of the Witwatersrand, Private Bag 3, Wits, 2050, Republic of South Africa

${ }^{d}$ Department of Chemistry, Vaal University of Technology, Private Bag X021, Vanderbijlpark, 1900, Republic of South Africa

$\dagger$ Electronic supplementary information (ESI) available. See DOI: 10.1039/c6ra00262e the ratio of the concentration of reagents to that of surfactants provides another control over NC size, since high stabilizer-toreagent concentrations favour the formation of more small nuclei initially and thus a smaller NC size., ${ }^{\mathbf{1 , 8}}$

With some of the above adjustments, colloidal method of synthesis have been reported to produce 2D nanosheets directly in solution, normally for compounds that assume layered crystal structures such as $\mathrm{SnSe}^{9,10}$ and in our case InSe. This is achieved by using surface stabilizing ligands that promote lateral growth while shortening vertical growth along the stacking axis of the crystals. ${ }^{\mathbf{9}, \mathbf{1 0}}$ This route of synthesis also permits the fundamental studies on dimension-dependent physical properties in exotic 2D systems like the colloidal nanosheets. ${ }^{11}$ Therefore, it is important for precise control of the characteristic features of the colloidal nanosheets such as their faceted edge dimensions, lateral uniformity and layer thickness which allow one not only to observe striking and unique nanocrystal properties but also to tune their chemical and physical properties as desired during fabrication. For instance, the band gap of these nanosheets can be tuned by changing the layer thickness thus offering opportunities for innovative device architectures in nanoelectronics and optoelectronics. $^{12}$

InSe is a group III-VI compound semiconductor that adopts a basic layered structure conforming to that of the $\gamma$-poly-type GaSe (rhombohedral modification) with a primitive unit cell that extends in two dimensions over three layers. ${ }^{13-17}$ It has direct band gap in bulk, anisotropic electronic properties and 
has potential application on photovoltaics. ${ }^{17-23}$ In addition, mechanically exfoliated InSe flakes have been effectively used as photodetectors with good response and quantum efficiency. ${ }^{24}$ Herein we report the successful colloidal synthesis of rhombohedral indium monoselenide (InSe) nanostructures more specifically the 2D nanosheets. During the synthesis, we investigated the effect of the indium concentration on the formation of perfect InSe nanosheets by monitoring the evolution of shapes with time. The peculiarity of this method was that we were able to produce via wet chemical synthesis, InSe nanosheets with properties that were comparable to those of the exfoliated InSe flakes in some of the previously reported studies as shall be discussed in the Results section. Also the function of oleylamine (OLA) in the formation of the InSe nanosheets was established during the reaction by introducing different dosages of 1-dodecanthiol (1-DDT) as a co-surfactant.

\section{Experimental procedures}

\subsection{Chemicals}

Indium(III) chloride, 99\% selenium powder, 70\% oleylamine (OLA), 98\% 1-dodecanthiol (1-DDT), chloroform and 96\% ethanol were obtained from Sigma Aldrich.

\subsection{Synthesis of InSe nanostructures}

One pot containing a mixture of $1.1059 \mathrm{~g}(5 \mathrm{mmol})$ of $\mathrm{InCl}_{3}$, $0.3948 \mathrm{~g}$ ( $5 \mathrm{mmol})$ of selenium in the case of mole ratio $1: 1$ \} and $10 \mathrm{ml}$ of OLA was quickly heated to a temperature of $200{ }^{\circ} \mathrm{C}$ under strong magnetic stirring and constant flow of nitrogen gas. At this temperature, aliquots were obtained after $8 \mathrm{~min}, 10$ $\mathrm{min}, 30 \mathrm{~min}$ and $60 \mathrm{~min}$. The aliquots were left to cool to $80{ }^{\circ} \mathrm{C}$ after which ethanol was added to crush out and also to wash the nanoparticles. The nanoparticles were then collected by centrifugation at $3000 \mathrm{rpm}$. The washing with ethanol was done several times in order to remove any excess reactants and surfactants. We then considered three other different mole ratio of $\mathrm{InCl}_{3}$ : Se following the same procedure. For $1: 1,2: 1$ and $3: 1$, the concentration of the In-precursor was varied and that of selenium was kept constant while vice versa was used in the case of the mole ratio $1: 2$. The synthesis of $\operatorname{In}_{2} \mathrm{Se}_{3}$ nanocrystals is described in the ESI. $\dagger$

\subsection{Instrumentation}

Optical characterization. A Varian Cary Eclipse (Cary 50) UVVis spectrophotometer was used to carry out the absorption measurements. A Varian Cary Eclipse EL04103870 fluorescence spectrophotometer with a medium PMT voltage at an excitation wavelength of $200 \mathrm{~nm}$ was used to measure the photoluminescence of the particles. For both spectral analysis, the powders were dissolved in chloroform and placed in quartz cuvettes ( $1 \mathrm{~cm}$ path length).

X-ray diffraction. XRD patterns on powdered samples were measured on a Bruker MeasSrv (D2-205530)/D2-205530 diffractometer using secondary graphite monochromated $\mathrm{CoK} \alpha$ radiation $(\lambda 1.78897 \AA)$ at $30 \mathrm{kV} / 30 \mathrm{~mA}$. Measurements were taken using a glancing angle of incidence detector at an angle of $2^{\circ}$, for $2 \theta$ values over $10-90^{\circ}$ in steps of $0.026^{\circ}$ with a step time of 37 $\mathrm{s}$ and at a temperature of $25^{\circ} \mathrm{C}$.

Electron microscopy. The transmission electron microscopy (TEM) was carried out on a FEI Tecnai T12 TEM microscopy operated at an acceleration voltage of $200 \mathrm{kV}$ with a beam spot size of $20-100 \mathrm{~nm}$ in TEM mode. The HRTEM images were obtained from a JEOL JEM-2100 microscopy with a LAB6 filament and an EDS detector, operated at $200 \mathrm{kV}$. The samples were prepared by placing a drop of the suspended nanoparticles in chloroform, on a carbon-copper grid. The grid with the sample was then allowed to dry at room temperature.

\section{Results and discussion}

\subsection{Morphological properties}

In order to study the formation process of the InSe nanosheets, concentration and time experiments were carried out and their formation mechanism was studied. From the TEM analyses, it was realized that perfect nanosheets were generated at a particular optimum reaction time, and that these durations varied for each of the three concentrations used, i.e., $8 \mathrm{~min}$ for $1: 1$, and $60 \mathrm{~min}$ for $2: 1$ and $30 \mathrm{~min}$ for $3: 1 \mathrm{In}:$ Se mole ratios as depicted in Fig. $1 * \mathrm{a},{ }_{\mathrm{f}}$ and ${ }^{*} \mathrm{~h}_{1}$ respectively. However, these perfect nanosheets were seen to possess a lot of similarities despite the difference in the reaction conditions (change in the concentration of the In-precursor and time of synthesis). For instance, they all showed regular faceted edges with six sides close to being hexagonal, though the sides were established to be slightly different by a few nanometres. In addition, the nanosheets portrayed different shapes within the same sample as illustrated on the insets in Fig. $1 * a, * f$ and ${ }^{*} h_{2}$, though all were seen to have lateral dimension of approximately $130 \mathrm{~nm}$. The nanosheet was slightly folded in Fig. $1 * \mathrm{~h}_{2}$ and transparent as seen in the inset, indicating that they are thin. To confirm this, the thicknesses of the nanosheets were established by the digital micrograph technique described in the ESI in Fig. S1 $\dagger$ and the results are summarized in Table $\mathrm{S} 1 . \dagger$ The approximated thicknesses were $18.3 \mathrm{~nm}$ for $1: 1,28.9 \mathrm{~nm}$ for $3: 1$ and $61.7 \mathrm{~nm}$ for $2: 1$. The difference in the thickness suggest that increasing reaction time as seen in the case of $2: 1$ mole ratio resulted in number of layers stacked on top of one another. It is worth mentioning that after the optimum time of synthesis for each concentration, the nanosheets begin to disintegrate into smaller nanoparticles as depicted in Fig. 1b and is a phenomenon reported in our previous study. ${ }^{7}$ Also, from Fig. $1 \mathrm{a}$ and b for $2: 1$ and $1 \mathrm{~g}$ for $3: 1$, the nanosheets produced showed indefinite shapes with irregular faceted edges, an indication that nanosheets were not fully developed at reaction times lower than the optimum. As a control study, an increase in the Se concentration did not result in nanosheets formation as portrayed in Fig. 1c regardless of the reaction time.

There are competing arguments in literature as to how the nanosheets form however evident from all the studies including ours, it is difficult to pin point the exact mechanism of formation of the nanosheets. ${ }^{25-29}$ Nevertheless, the formation of two dimensional InSe nanosheets may be explained by the mechanism reported by Lu et al. ${ }^{28}$ The mechanism suggests that 
1:1
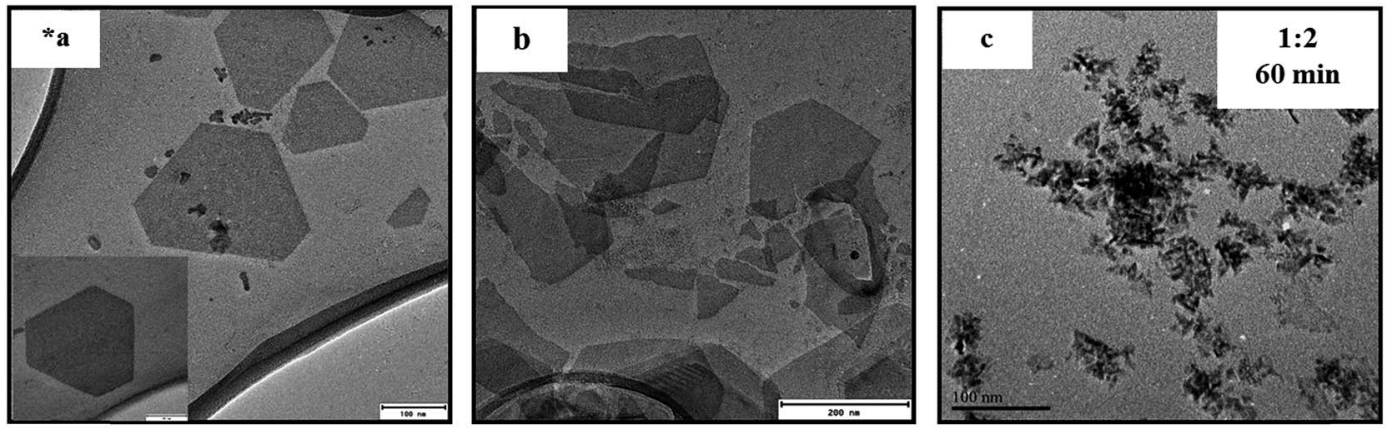

2:1
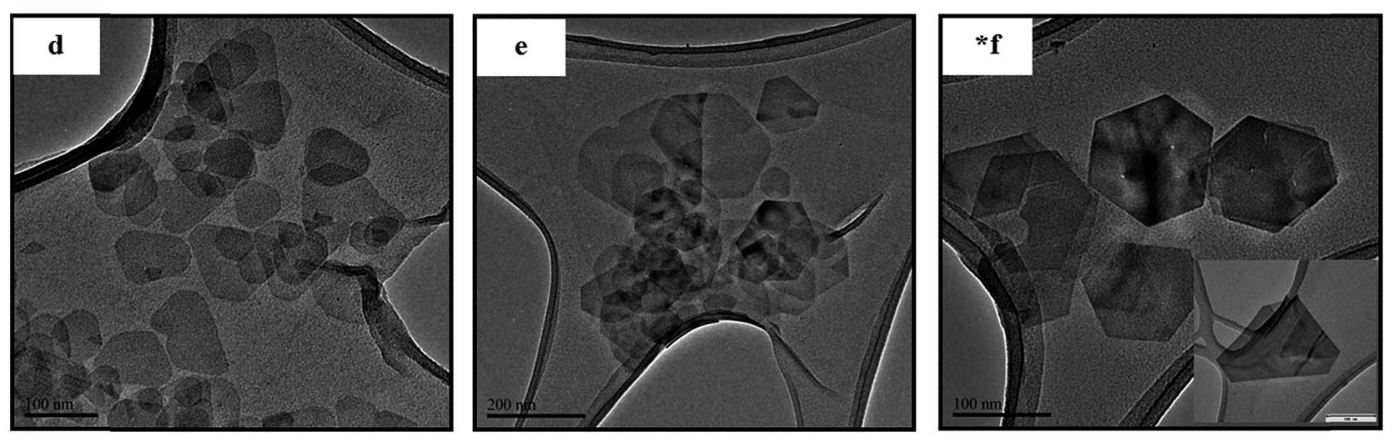

$3: 1$
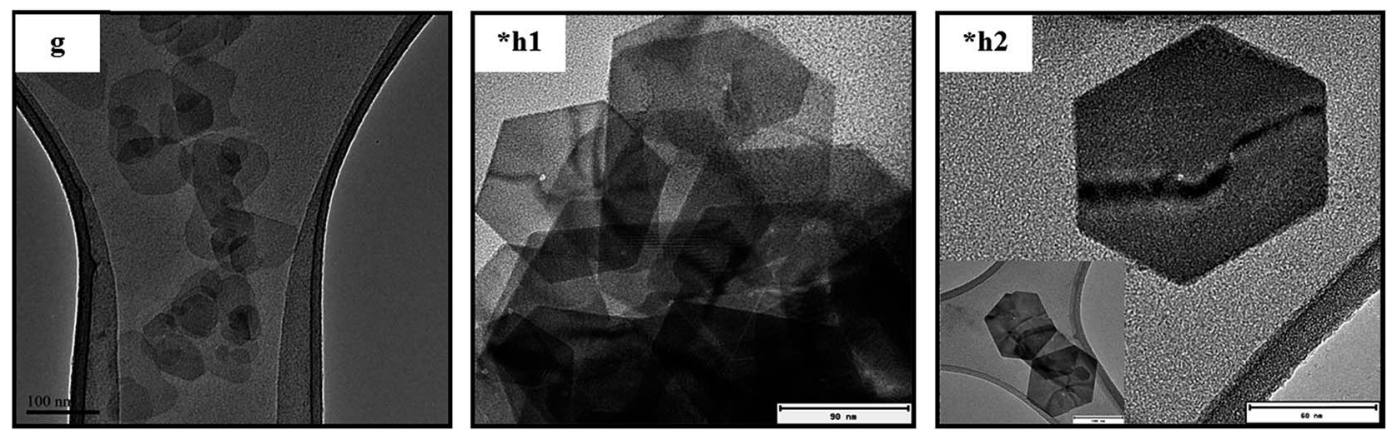

Fig. 1 TEM images of nanostructures obtained by varying the mole ratio $\left(\mathrm{InCl}_{3}: \mathrm{Se}\right)$; (a and b) were synthesized at $1: 1$ for 8 min and 10 min respectively, (c) was synthesized at 60 min mole ratio $1: 2$. For (d-f) synthesis was done at mole ratio $2: 1$ at 8,30 and 60 min respectively. Images (g) $8 \mathrm{~min}$ and $\left(\mathrm{h}_{1}\right.$ and $\left.\mathrm{h}_{2}\right) 30 \mathrm{~min}$ are of samples obtained at a mole ratio $3: 1$. The * shows the optimum time when perfect nanosheets can be obtained at different mole ratio, i.e. for $1: 1$ - optimum time is $8 \mathrm{~min}, 2: 1$ is $60 \mathrm{~min}$ and $3: 1$ is 30 min. $1: 2$ does not produce nanosheets at any time.

nanosheet formation can be elucidated by the anisotropic nature of the crystal structure of the layered materials coupled with the type of coordinating solvent used during synthesis. Thus as shown in Scheme 1a, the $\gamma$-InSe polytype crystalline structure of InSe contains layers of covalently bonded In-Se and In-In bonds. The orientation of the atoms in each layer is such that the orbitals of the valent electrons involved in bond formation are located inside the layer. Therefore there is no chance of an overlap with orbitals of the valent electrons from the neighbouring layer. ${ }^{30}$ As a result, these layers are held together through van der Waals bonds and stack vertically along the $c$-axis of the crystal structure. This then allows for intercalation of the layered crystal by foreign atoms or molecules as shown in Scheme 1b. When InSe begins to form in solution, the coordinating solvent (in this case OLA) infiltrates into the van der Waal space as it tries to binds to the nanocrystal, thereby preventing growth along the $c$-axis and supporting growth within the $a-b$ plane to form two-dimensional nanosheets.

HRTEM was done on the perfect nanosheets prepared after 8 $\min$ for mole ratio $1: 1,60 \mathrm{~min}$ for $2: 1$ and $30 \mathrm{~min}$ for $3: 1$ and the images are represented in Fig. 2a-c.

They all showed high crystalline structure with lattice spacing of $3.3 \AA$ which is consistent with the $d$-spacing obtained from the XRD. The intensity peaks from the selected area electron diffraction formed six sided patterns that have been observed in $\mathrm{GaSe}^{31}$ and InSe flakes. ${ }^{12}$ The lattice fringes as shown in the high magnification inset in Fig. 2d formed a hexagonal patterned structure that resembled a fourfold 

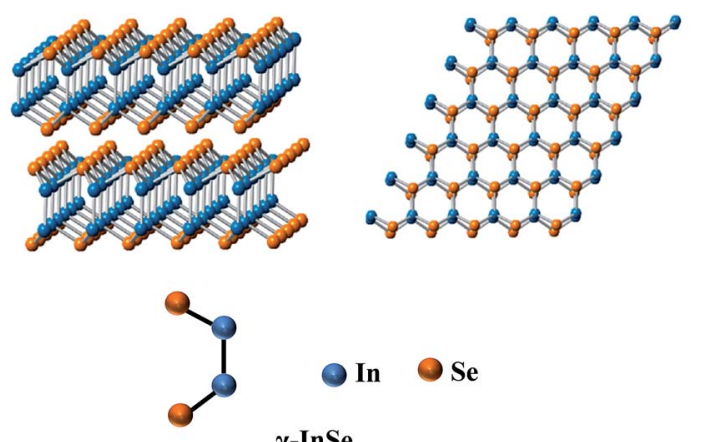

b
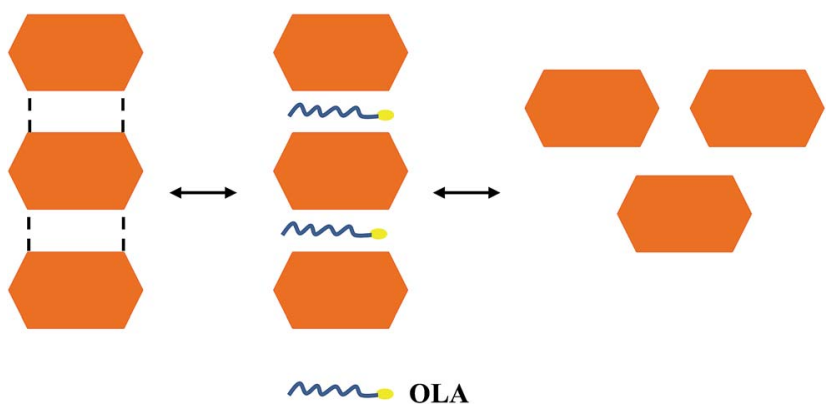

Scheme 1 (a) Three dimension bulk crystal structure of InSe containing layers that are held together by van der Waal's forces stack vertically along the $c$-axis and (b) the proposed mechanism for the formation of nanosheets.
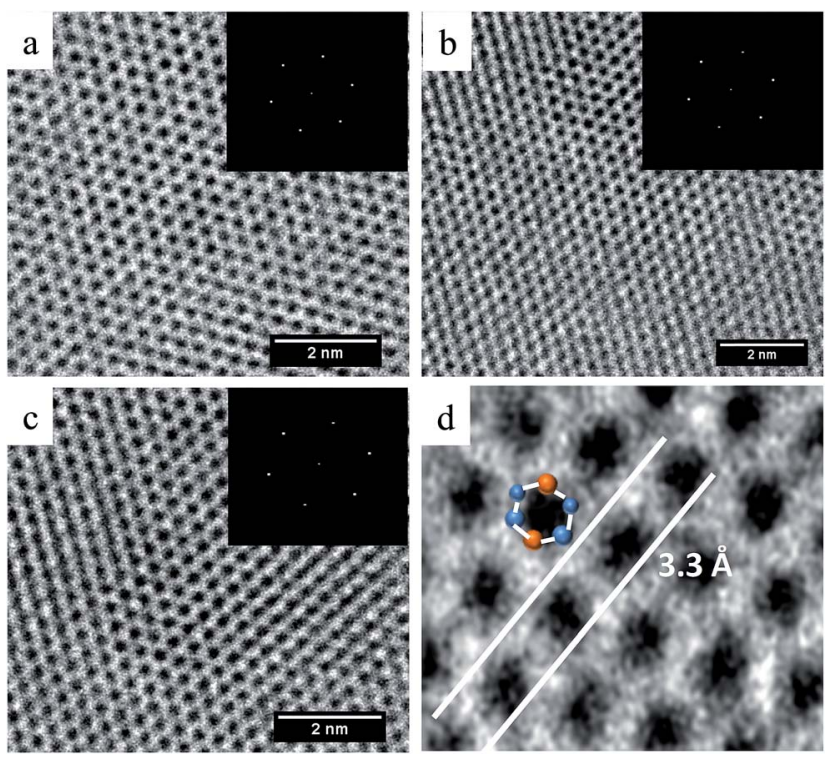

Fig. 2 HRTEM of perfect nanosheets produced at (a) 8 min $1: 1$, (b) 30 $\min 3: 1$ (c) $60 \min 2: 1$ respectively. The inset on each shows the selected area diffraction patterns that are indication of the crystallinity nature of the nanosheets. Fig. $3 d$ shows a part of the HRTEM image at higher magnification for the $60 \mathrm{~min} 2: 1$. It depicts the hexagonal pattern formed on the crystal structure of the nanosheet.

stacking of Se-In-In-Se monoatomic sheets. This is in agreement with the basic layered structure of bulk InSe which has a primitive unit cell that extends over three layers and within each layer, a fourfold stacking of Se-In-In-Se monoatomic sheets with a hexagonal structure as represented in Scheme 1a. ${ }^{4}$

The HRTEM images also had similar features, an indication that the end product (nanosheets) obtained when the concentration of In-precursor was varied yielded a $1: 1$ stoichiometry of In : Se regardless of the variation and time. This therefore depicted an independency of the stoichiometry with concentration or time but the morphology did reveal interdependency between concentration and time on the formation of the nanosheets albeit with slightly different facets. This is illustrated in Scheme 2.

XRD analysis (Fig. 3a) was used to verify the phase structure and composition of the nanosheets obtained at the optima time of syntheses for the three mole ratio; $8 \mathrm{~min}$ for $1: 1,60 \mathrm{~min}$ for $2: 1$ and 30 min for $3: 1$ and they are represented by spectra $\mathrm{x}, \mathrm{y}$ and $\mathrm{z}$ respectively. The relative similarity in the spectra was an indicator that the change in the concentration of the indium precursor yielded nanosheets with the same chemical composition. The crystalline structures of these nanosheets were predominantly rhombohedral InSe phase (JCPDS card 03-065$3660)$ as all the peaks were indexed to this phase. The lattice parameters were $a=4 \AA$ and $c=24.32 \AA$ with a $d$-spacing of 3.33 $\AA$ along 101 planes. The values for the lattice parameters are comparable to the ones obtained by Mudd $e t a l .^{12}$ as well as those reported by Rigoult et al. ${ }^{32}$ The difference in the intensity of the diffraction peak is relatively small and this may be an indication that some nanosheets were more crystalline. For

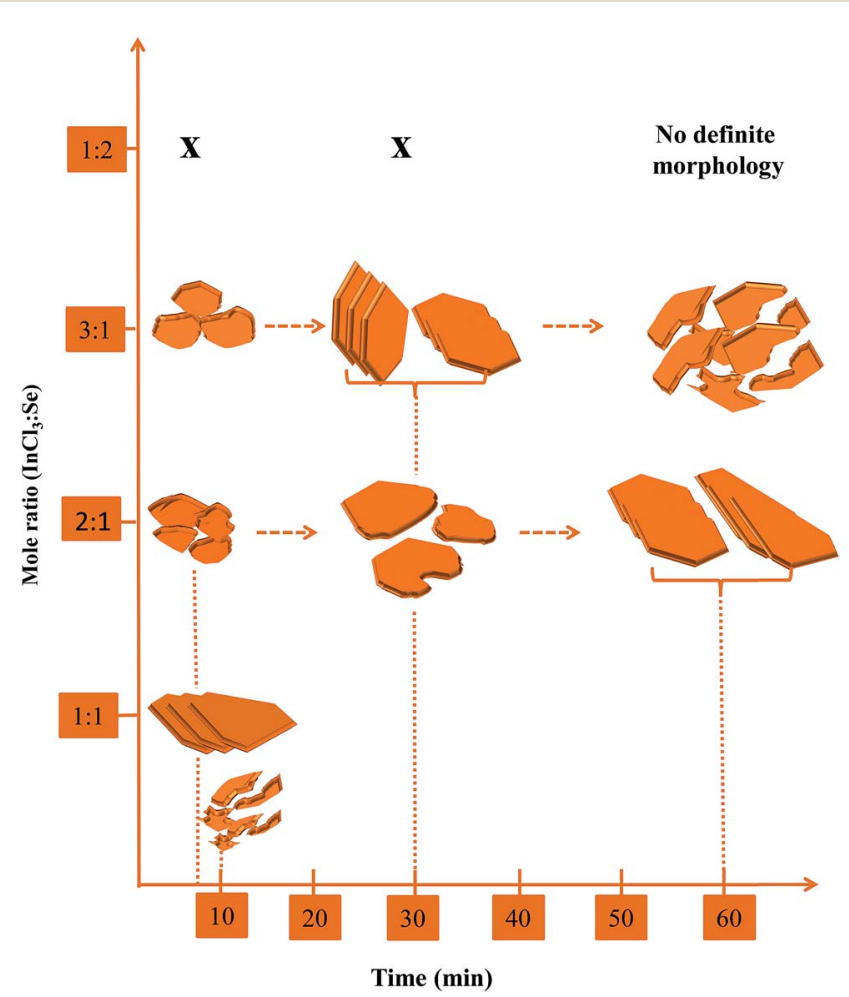

Scheme 2 Representation of different nanostructures obtained at different reaction times with a variation of concentration of the metal precursors. The $\mathrm{X}$ indicates the absence of nanostructures at those conditions. 


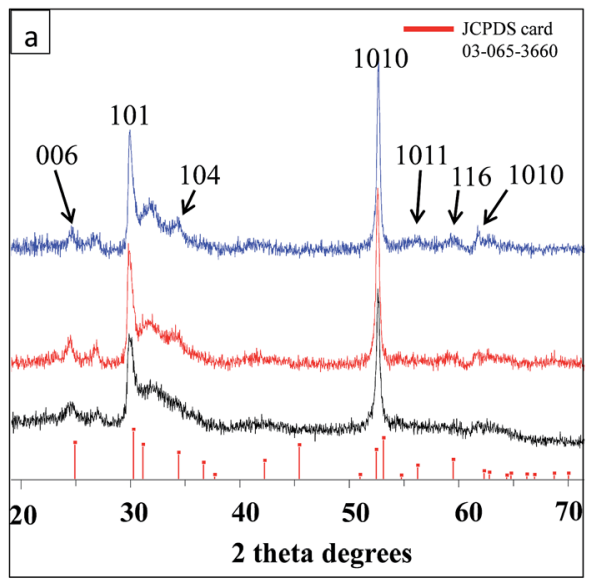

b

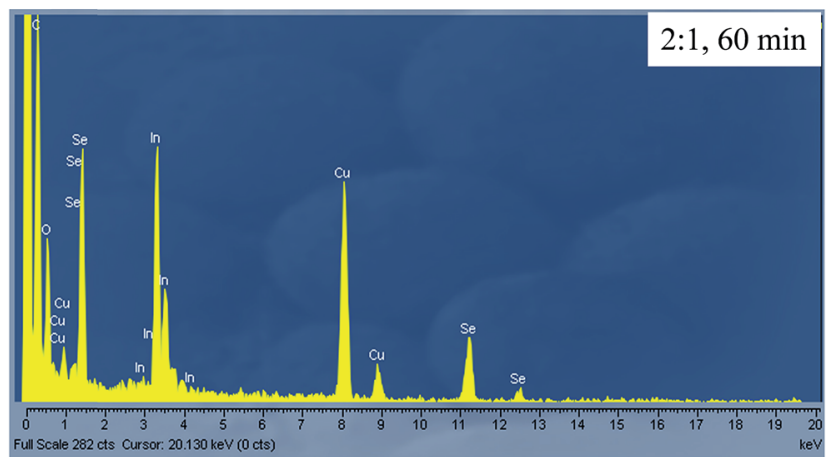

Fig. 3 (a) XRD patterns for the nanosheets obtained at each optimum time of synthesis for the three mole ratios, $\operatorname{lnCl}_{3}$ : $\mathrm{Se}$; (x) 8 min for $1: 1$, (y) $30 \mathrm{~min}$ for the ratio $3: 1$ and (z) $60 \mathrm{~min}$ for the mole ratio $2: 1$ respectively, (b) energy dispersive spectrum of the sheets obtained after 60 min with a mole ratio of $2: 1$.

instance the intensity of the $3: 1$ mole ratio $\{\mathrm{z}\}$ appeared less intense compared to spectra $\{x\}$ and $\{y\}$. The composition of the nanosheets obtained after $60 \mathrm{~min}$ using a mole ratio of $2: 1$ were further examined by energy dispersive spectroscopy (EDS) (Fig. $3 b$ ). The $L \alpha$ peaks of indium and the selenium confirmed that the nanosheets were composed of indium and selenium. The copper and carbon peaks were from the copper grid that was used during the analysis.

From the above investigations on the formation of InSe nanosheets, it is apparent that despite the increase in concentration of the indium precursor, nanosheets with comparable properties are produced at different reaction times. This has been attributed to the use of OLA as both a coordinating solvent and a reducing agent. Therefore, in order to understand the role of OLA in the shape evolution and properties of the final $\operatorname{In}_{x} \mathrm{Se}_{y}$ nanocrystals, we decided to follow the work done by Ramasamy et al. ${ }^{33}$ Typically, different amounts of 1-DDT were added as a cosurfactant to the reaction mixture containing $5 \mathrm{mmol}$ of $\mathrm{InCl}_{3}$ and $5 \mathrm{mmol}$ of Se maintaining the procedure used before (method and figures in the ESI $\dagger$ ).

Based on the above results and that in Fig. S1, $\uparrow$ a possible mechanism explaining the function of OLA in the formation of $\mathrm{In}_{x} \mathrm{Se}_{y}$ nanocrystals is proposed. OLA has been reported to not only act as a solvent in many organic and inorganic syntheses, but also it can be a surfactant and even a mild reducing agent, depending on the reaction conditions and the nature of the target nanocrystals. ${ }^{34}$ For example, Park et al..$^{35}$ and Chen et al. ${ }^{36}$ used OLA as a stabilizer and a solvent during their synthesis of $\beta$ - $\operatorname{In}_{2} \mathrm{~S}_{3}$ at $215^{\circ} \mathrm{C}$. In other studies, the concentration of OLA as a co-ligand affected the composition and the size of the nanocrystals obtained..$^{37}$ In addition, in the presence of a stronger reducing agent, the role of OLA is limited to act as a surfactant and/or solvent. ${ }^{38}$ In our case, when OLA is used as the sole coordinating solvent and a mild reducing agent, $\mathrm{In}^{3+}$ ions are reduced to $\mathrm{In}^{2+}$ ions and the elemental selenium reduced to $\mathrm{Se}^{2-}$ ions thus resulting in the formation of InSe. This is illustrated in Scheme 3a and it was experienced in all the three concentration used in the formation of InSe nanosheets regardless of the amount of the In-precursor added, thus explaining the existence of one particular morphology of the obtained nanocrystals with comparable characteristics. On the other hand, the presence of 1-DDT in the reaction mixture resulted in the stabilization of the $\mathrm{In}^{3+}$ ions through the formation of an In-DDT complex while the selenium was reduced by the OLA to $\mathrm{Se}^{2-}$. Scheme $3 \mathrm{~b}$ elaborates the interaction of $\mathrm{In}^{3+}$ and $\mathrm{Se}^{2-}$ ions to form $\mathrm{In}_{2} \mathrm{Se}_{3}$ nanostructures. Another suggestion from the reaction environment containing 1-DDT was that $50 \%$ OLA $(5 \mathrm{ml}$ OLA $+5 \mathrm{ml} 1$-DDT) produced agglomerated products. This was due to the insufficient reducing agent to reduce the elemental selenium thus introducing impurities during the reaction hence the poor morphology unlike when $80 \%$ of oleylamine was used which resulted in the nanowires.

$100 \%$ of Oleylamine (OLA)

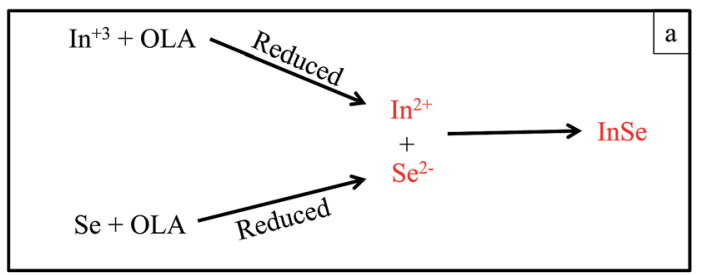

$80 \%$ OLA $+20 \% 1$-DDT and $50 \%$ OLA $+50 \% 1$-DDT

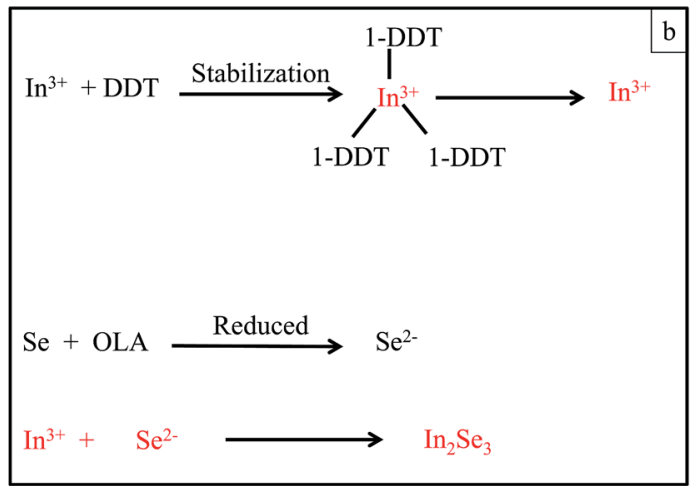

Scheme 3 Schematic representation of (a) reduction process $\ln ^{3+}$ to $\mathrm{In}^{2+}$ and $\mathrm{Se}$ to $\mathrm{Se}^{2-}$ when $100 \%$ oleylamine was used and (b) the stabilization process of $\mathrm{In}^{3+}$ ions by both $2 \mathrm{ml}$ and $5 \mathrm{ml}$ of 1-DDT introduced in the reaction mixture. 


\subsection{Optical studies of the nanosheets}

To further understand the properties of the nanosheets, optical studies were done by analysing their optical absorption and emission spectra. Examination of the absorption spectra displayed in Fig. 4a revealed that the nanosheets exhibited quantum confinement effects, that is, their absorption band edges were blue shifted compared to that of bulk InSe which is about $1000 \mathrm{~nm}^{39}$ The nanosheets obtained at 8 min 1:1 (Fig. 4a spectrum $\mathrm{x}$ ) presented a tailing end demonstrating that the sample had nanosheets of different sizes as confirmed by TEM image in Fig. 1a. However, there was a slight bend around $724 \mathrm{~nm}$ giving an approximate value of the band edge. The nanosheets produced at $60 \mathrm{~min} 2: 1$ (Fig. 4 a spectrum y) and 30 min $3: 1$ (Fig. 4 a spectrum $\mathrm{z}$ ) also had their band edges at about $725 \mathrm{~nm}$. These values are seen to be similar, indicating that the absorption onset was not affected by the change in the mole ratio when developing InSe nanosheets and that nanosheet with relatively same properties were obtained as suggested by XRD results.

The corresponding band scheme of bulk InSe is represented in Fig. $4 b^{11,39}$ and it is used herein to make emphasis on the observation made from the PL emission spectra of the nanosheets. The electronic transition from $\mathrm{p}_{x, y}$ like orbitals to the bottom of the conduction band are the $E^{\prime}$ transition $(2.4 \mathrm{eV})$ while the $\mathrm{p}_{z}$ like orbitals to the bottom of the conduction band is the band gap $(1.27 \mathrm{eV})$. Our measurements were performed at an excitation wavelength of $240 \mathrm{~nm}$ to provide energy greater than the band gap of InSe. This has been reported to allow the occurrence of the transition between $\mathrm{p}_{x, y}$ and the bottom of the conduction. ${ }^{39}$ It was observed that all the nanosheets synthesized at the three different optimum conditions gave two emission peaks (Fig. 4c) centred at position (I) due to the $E^{\prime}$ transition and (II) as a result of the band gap transition. In addition, the peak positions were blue shifted compared to $\mathbf{a}$

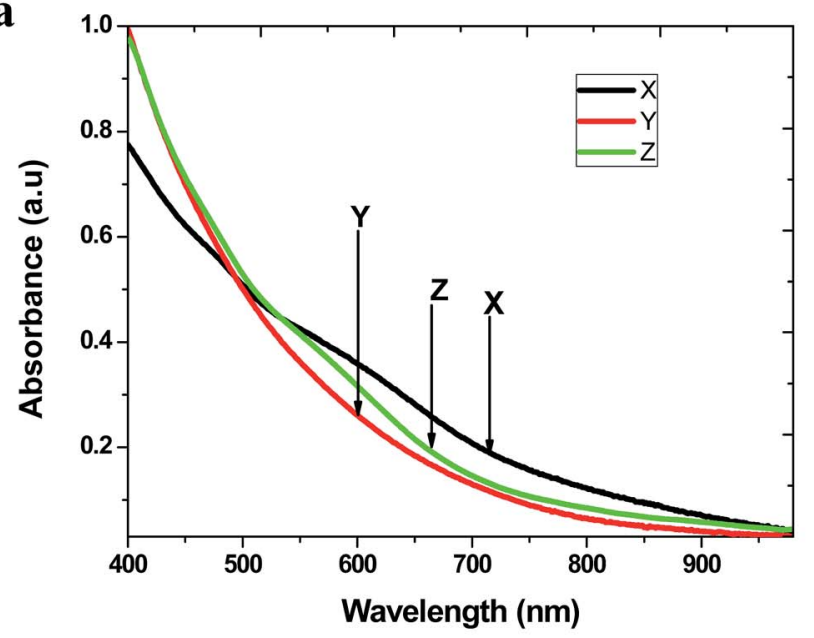

c

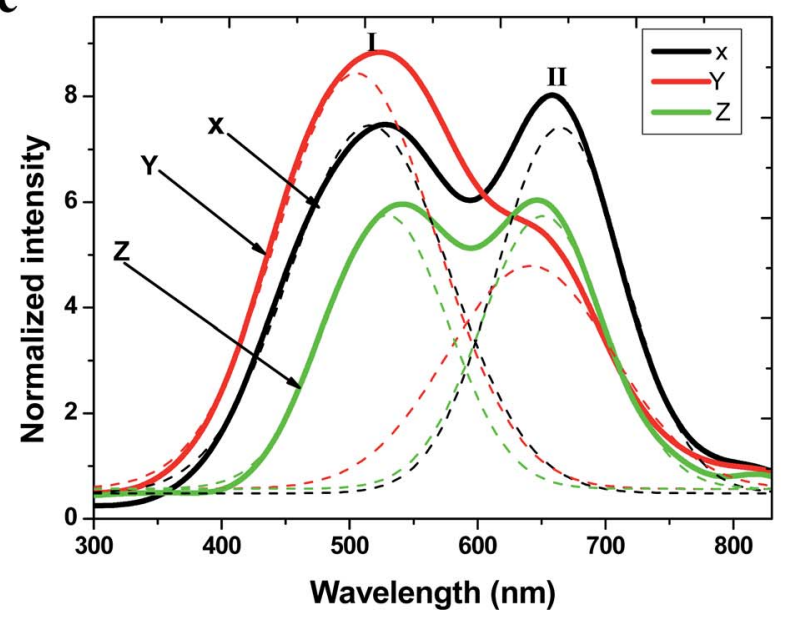

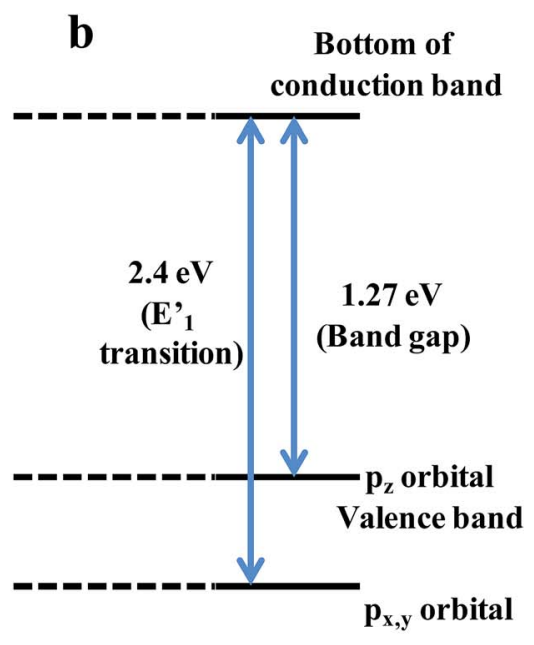
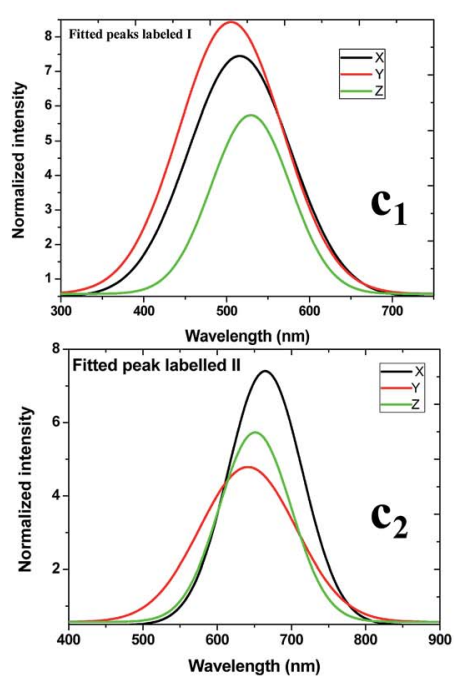

Fig. 4 (a) Absorption spectra of nanosheets synthesized at (x) $8 \min 1: 1$, (y) $60 \min 2: 1$ and (z) 30 min $3: 1$; all the mole ratios are InCl 3 : Se (b) the band structure of InSe with the bulk band gap and electronic transitions, and (c) the photoluminescence (PL) spectra of the nanosheets in the same order of synthesis as of the absorption spectra. $\left(\mathrm{C}_{1}\right)$ and $\left(\mathrm{C}_{2}\right)$ are the Gaussian fitted peaks for the regions (I) and (II) in the PL emission spectra emphasizing on the reduction on the intensity of the peaks. 
Table 1 The centre positions of the emission peaks for the perfect nanosheets from the PL emission spectra

\begin{tabular}{lll}
\hline Spectrum & $\begin{array}{l}\text { Emission peak at } \\
\text { I region (nm) }\end{array}$ & $\begin{array}{l}\text { Emission peak at } \\
\text { II region (nm) }\end{array}$ \\
\hline $\mathrm{x}$ & 519 & 657 \\
$\mathrm{y}$ & 515 & 664 \\
$\mathrm{z}$ & 520 & 651
\end{tabular}

their absorption band edges; a phenomena reported in the previous studies on InSe and GaSe nanosheets ${ }^{11,31}$ and other nanosheets obtained from transition metals dichalcogenides as band gap crossover due to the modification of the band structure caused by the decrease in layer thickness. ${ }^{40}$

Table 1 summarizes the centre positions of the emission peaks for the perfect nanosheets. According to Mudd et al., the PL intensity signal of InSe flakes decreases with a decreasing nanosheet thickness i.e. there is quenching of the PL intensity as a result of band gap crossover. ${ }^{12}$ However this trend is not observed in our study as illustrated by the fitted Gaussian peaks from the emission spectra represented in Fig. $4 c_{1}$ (I region) and $c_{2}$ (II region). Nevertheless the presence of two emission peaks is still a strong indicator of the band crossover phenomenon.

\section{Conclusions}

In summary, our investigations confirmed that the formation of $\mathrm{In}_{x} \mathrm{Se}_{y}$ nanocrystals is conceivable via a direct colloidal route. Hexagonal like InSe nanosheets were synthesized at different optimum times depending on the amount of the indium precursor. Also evident was the fact that regardless of the amount of the indium precursor used, the product remained unchanged i.e. InSe. This was attributed to the nature of the oleylamine capping agent. Oleylamine is known to be a reducing agent and in this case resulted in the reduction of $\mathrm{In}^{3+}$ to $\mathrm{In}^{2+}$ and elemental selenium to $\mathrm{Se}^{2-}$ thus always resulting in the formation of rhombohedral InSe 2D nanosheets. To consolidate this observation, a non-reducing 1dodecanthiol co-surfactant was used. DDT which is a much softer and polarized ligand than OLA was thought to stabilize the $\mathrm{In}^{3+}$ ion by coordinating and forming a complex thus preserving the +3 oxidation state of indium hence resulting in the formation of the final product of $\operatorname{In}_{2} \mathrm{Se}_{3}$ nanocrystals. The morphology of $\operatorname{In}_{2} \mathrm{Se}_{3}$ changed depending on the amount of OLA used. The optical properties of InSe were further evidence that 2D nanosheets had been synthesized due to the band gap crossover readily observed in 2D nanosheets.

\section{Acknowledgements}

The authors would like to thank the University of the Witwatersrand, School of Chemistry; the MMU particularly Prof A. Ziegler for the sheet thickness measurements and the X-Ray facility at Wits; Profs B. Mothudi and M. S. Dhlamini from UNISA for the fluorescence measurements, the National
Research Foundation (NRF), TESP and the Organization for Women in Science in Developing Countries (OWSD) for their funding.

\section{References}

1 C. B. Murray, S. Shouheng, W. Gaschler, H. Doyle, T. A. Betley and C. R. Kagan, IBM J. Res. Dev., 2001, 45(1), 47.

2 N. Moloto, M. J. Moloto, N. J. Coville and S. Sinha Ray, J. Cryst. Growth, 2011, 324, 41.

3 Y. Matsai, R. Polsky, Y. Koltypin, A. Gedanken and G. Hodes, J. Am. Chem. Soc., 1999, 121, 10047.

4 N. Pickett and P. O'Brien, Chem. Rec., 2001, 1, 467.

5 X. Peng, L. Manna, W. Yang, J. Wickham, E. Scher, A. Kadavanich and A. P. Alivisatos, Nature, 2000, 404, 59.

6 N. Moloto, N. Revaprasadu, P. L. Musetha and M. J. Moloto, J. Nanosci. Nanotechnol., 2009, 9(8), 4760.

7 M. A. Airo, S. Gqoba, P. K. Mubiayi, S. Govindraju, M. J. Moloto and N. Moloto, J. Cryst. Growth, 2014, 406, 1.

8 S. Sun, C. B. Murray and H. Doyle, Mater. Res. Soc. Symp. Proc., 1999, 577, 385.

9 D. D. Vaughnll, S.-L. In and R. E. Schaack, ACS Nano, 2011, 5(11), 8852.

10 I. T. Sines, D. D. Vaughnll, A. J. Biacchi, C. E. Kingsley, E. J. Popczun and R. E. Schaak, ACS Nano, 2012, 24, 3088.

11 O. Minoru and S. Takayoshi, Chemical nanomanipulation of two-dimensional nanosheets and its applications, ed. Y. Masuda, 2011, ISBN: 978-953-307-912-7, http:// www.intechopen.com.

12 G. W. Mudd, S. A. Svatek, T. Ren, A. Patane, O. Makarovsky, L. Eaves, P. H. Beton, Z. D. Kovalyuk, G. V. Lashkarev, Z. R. Kudrynskyi and A. I. Dmitriev, Adv. Mater., 2013, 25, 5714.

13 J. Camassel, P. Merie and H. Mathieu, Phys. Rev. B: Condens. Matter Mater. Phys., 1978, 17(12), 4719.

14 R. H. Williams, J. V. McCannyt, R. B. Murray, L. Ley and P. C. Kemenygll, J. Phys. C: Solid State Phys., 1977, 10, 1223.

15 S. Tyrrell, M. Swadźba-Kwaśny and P. Nockemann, J. Mater. Chem. A, 2014, 2, 2616.

16 X. Tan, J. Zhou and Q. Yang, CrystEngComm, 2011, 13, 2792. 17 N. Revaprasadu, M. Azad Malik, J. Carstens and P. O'Brien, J. Mater. Chem., 1999, 9, 2885.

18 M. Millot, J. M. Broto, S. George, J. Gonzalez and A. Segura, Phys. Rev. B: Condens. Matter Mater. Phys., 2010, 81, 205211.

19 A. I. Dmitriev, V. V. Vishnjak, G. V. Lashkarev, V. L. Karbovskyi, Z. D. Kovaljuk and A. P. Bahtinov, Phys. Solid State, 2011, 53, 622.

20 R. Sreekumar, R. Jayakrishnan, C. S. Kartha and K. P. Vijayakumar, J. Appl. Phys., 2008, 103, 023709.

21 S. T. Lakshmikumar and A. C. Rastogi, Sol. Energy Mater. Sol. Cells, 1994, 32, 7.

22 A. Segura, Sol. Energy Mater., 1979/1980, 2, 159.

23 A. Segura, J. P. Guesdon, J. M. Besson and A. Chevy, J. Appl. Phys., 1983, 54, 876.

24 S. Lei, L. Ge, S. Najmaei, A. George, R. Kappera, J. Lou, M. Chhowalla, H. Yamaguchi, G. Gupta, R. Vajtai, A. D. Mohite and P. M. Ajayan, ACS Nano, 2014, 8, 1263. 
25 Z. Li and X. Peng, ACS Nano, 2011, 133, 6578.

26 J. S. Son, J. H. Yu, S. G. Kwon, J. Lee, J. Joo and T. Hyeon, Adv. Mater., 2011, 23, 3214.

27 K. D. Oyler, X. Ke, I. T. Sines, P. Schiffer and R. E. Schaak, Chem. Mater., 2009, 21, 3655.

28 W. G. Lu, Y. Ding, Y. X. Chen, Z. L. Wang and J. Y. Fang, J. Am. Chem. Soc., 2005, 127(28), 10112.

29 G. Q. Zhang, W. Wang, X. L. Lu and X. G. Li, Cryst. Growth Des., 2009, 9(1), 145.

30 Y. Zhirko, V. Trachevsky and Z. Kovalyuk, Hydrogen storage: on the possibility of layered crystals application for solid state hydrogen storages-InSe and GaSe crystals, INTECH Open Access Publisher, 2012, pp. 211-242.

31 P. Hu, Z. Wen, L. Wang, P. Tan and K. Xiao, ACS Nano, 2012, 6(7), 5988.

32 J. Rigoult, A. Rimsky and A. Kuhn, Acta Crystallogr., Sect. B: Struct. Crystallogr. Cryst. Chem., 1980, 36, 916.
33 P. Ramasamy, P. Manivasakan and J. Kim, CrystEngComm, 2015, 17, 807.

34 S. Mourdikoudis and L. M. Liz-Marzán, Chem. Mater., 2013, 25, 1465.

35 K. H. Park, K. Jang and S. U. Son, Angew. Chem., Int. Ed., 2006, 45, 4608.

36 B. Chen, S. Chang, D. Li, L. Chen, Y. Wang, T. Chen, B. Zou, H. Zhong and A. L. Rogach, Chem. Mater., 2015, 27, 5949.

37 H. Yang, N. Fan, W. Luan and S. Tu, Nanoscale Res. Lett., 2009, 4, 344.

38 J. Joo, H. Bin Na, T. Yu, J. H. Yu and Y. W. Kim, ACS Nano, 2003, 125, 11100.

39 A. Segura, J. Bouvier, M. V. Andrés, F. J. Manjón and V. Muňoz, Physical Review B-American Physical Society, 1997, 56(7), 4075.

40 S. Tongay, J. Zhou, C. Ataca, K. Lo, T. S. Matthews, J. Li, J. C. Grossman and J. Wu, Nano Lett., 2012, 12, 5576. 